\title{
Modeling and Control of an Aerial Multi-Cargo System: Robust Acquiring and Transport Operations
}

\author{
J. Castillo ${ }^{1,2}$, J. Escareno ${ }^{3}$, I. Boussaada ${ }^{1,2,4}$, O. Labbani ${ }^{3}$ and K. Camarillo ${ }^{5}$
}

\begin{abstract}
In this paper, we introduce the concept of a multilink unmanned aerial system (ML-UAS) designed for multicargo transportation tasks. Such a system features three links who are actuated by four flying robots. We present in detail the dynamics modeling based on the Euler-Lagrange formulation. A preliminary control scheme based on robust Adaptive Integral Sliding Mode Control (AISMC) is applied considering the MLUAS model uncertainties and external disturbances. The control objective is to follow/track avian-inspired acquiring patterns for acquiring operations. The effectiveness of the proposed strategy is validated by numerical simulations.
\end{abstract}

\section{INTRODUCTION}

In recent years, Unmanned Aerial Vehicles (UAVs) have been used for different tasks in the industrial or the scientific field. Recently, UAVs applications have been evolving specially those involving aerial environment-interactivity (manipulation, assembling, picking, transport) it is also the case for swarm-based cooperation which demands a high degree of dexterity [1].

Aerial robots have been extensively used for natural disaster assessment. In this mission context, the contribution of micro aerial vehicles (MAVs) remains restricted to the collection of images either in hovering or in-motion flight. Thus, the vehicles capable of picking, transporting and placing represent an ideal solution for survival-kit delivering, rescue operation, sensor deployment/acquiring. The impact of these capabilities might be amplified whether we multiply the aerial robots and develop efficient interaction algorithms.

\section{A. Related work}

There exist different approaches and strategies as well as a variety of configurations to address the the multi-vehicles interaction problem. Inspired by parallel manipulators, [2] presents a flying robot composed of three off-the-shelf quadcopters rigidly attached via an articulated structure. The implementation aerial system, which comprises three links and a platform, yielded to interesting results showing the system's dexterity as well as enhanced cargo capacity.

The necessity to increase efficiency flight raises the interest in transformable/morphing flying robots. In [3] the problems of flight stability and center of gravity shifting due to the payloads motion are studied. In this regard, [4] exposes a

\footnotetext{
${ }^{1}$ LS2A IPSA, Ivry-sur-Seine, France. jose. castillo@ipsa. fr

2 L2S, Université Paris Sud-CNRS-CentraleSupelec, Université Paris Saclay, Gif-sur-Yvette, France

3 XLIM UMR CNRS 7252, Université de Limoges, Limoges, France

${ }^{4}$ Inria Saclay, Equipe DISCO

5 Mechanical Engineering Department, Tecnológico Nacional de México en Celaya, Celaya, Mexico
}

multi-rotor aerial vehicle with two-dimensional multi-links and demonstrates stable aerial transformation for high mobility in three-dimensional environments. The DRAGON transformable aerial robot is introduced in [5]. This flying machine is a dual-rotor-embedded multi-link robot with the ability of multi-degree of freedom aerial transformation and the full pose control regarding the center of gravity of multi-links.

The task of manipulation several cargos implies the understanding of the phenomena present in transporting a single object which is a case widely studied in the literature. Hereof, the implementation of Kalman Filter to estimate the payloads disturbances [6] and the trajectory path picking generation in windy environments [7] can be cited, along with the use of learning automatas [8] and of fuzzy logic for computing the effects of the cargos in several quadrotors [9].

Nonetheless, the control by the means of the $H_{\infty}$ control theory has been adopted due to its robustness [10]. By combining the $\mathrm{H}_{2}$ and $H_{\infty}$ controls, [11] solves the trajectory tracking problem of a tilt-rotor UAV when transporting a suspended load.

The Sliding Mode Control (SMC) is another robust strategy control applied to aerial vehicles as in [7], [12] or [13].

\section{B. Paper contribution}

This paper presents the detailed longitudinal modeling and robust control of a novel multi-link aerial interactive system that is studied for multi-object acquisition and transport tasks. The Euler-Lagrage approach is used to deduce the equations of motion and couplings of the ML-UAS. The resulting expressions are rewritten for the sake of the control design. In terms of control, a Sliding Mode Controller alongside an adaptive gain with an integral term of the tracking error added to the sliding surface is designed (for details [13], [5], [7]). Furthermore, an extension to the multilink case of the avian-inspired trajectory generation scheme for aerial grasping is presented [7]. The latter is endowed with a links-dependant trajectory dynamics to generate a smooth pattern to achieve multi-object acquisition/placing.

\section{Problem Formulation}

In the regard of aerial picking and transportation, a single aerial rotorcraft is limited in dexterity and payload-carrying capacity. The employment of several rotorcrafts and the theory of multi-agent systems is often the emergent solution to the aforementioned issues despite the interaction difficulties and constrains [1], [4]. 


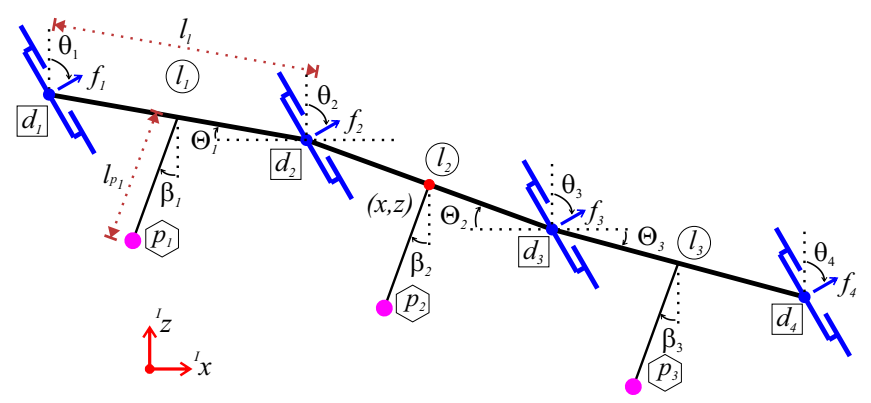

Fig. 1. Multi-linked Unmanned Aerial System description

A flying kinematic chain inspired by the capabilities of aerial vehicles, the dexterity of arm manipulators and a train-like transporting operation is introduced herein to overcome the established problematic.

\section{Paper Outline}

The paper is organized as follows: Section I corresponds to the general description of the paper. Section II comprises the equations describing the kinematics and the dynamics of the chain. The mathematical motion description of the system is used in Section III to generate a robust control law to overcome the aforementioned problematic while the trajectory generation is fully described in Section IV. We validate our proposal by numerical simulations whose explanation and results are given in Section V. Finally, the conclusions and future work remarks are written in Section VI.

\section{MATHEMATICAL MODELLING}

Let us consider the 3 links and 4 rotorcrafts system as a flying kinematic chain and its lateral dynamics only as shown in Fig. 1.

The links are considered to be attached to the center of gravity of the corresponding vehicles and to have a frictionless angular motion since the due-to disturbances can be mitigated by the robust controller [2] [10] [13]. In addition, we assume that the links have the same length $l_{l} \in \mathbb{R}$ and mass $m_{l} \in \mathbb{R}$ and, as a consequence, the same moment of inertia $I_{l} \in \mathbb{R}$. The attitude of the $j-t h$ link is described by the angle $\Theta_{j} \in \mathbb{R}$ where $j=1,2,3$.

The attitude of the $i-t h$ rotorcraft (with $i=1,2,3,4$ ) is given by the angle $\theta_{i} \in \mathbb{R}$ and measured as shown in Fig. 1 . The masses of the vehicles are given as $m_{r} \in \mathbb{R}$ and their moments of inertia as $I_{r} \in \mathbb{R}$.

The pose of $j-t h$ pendulum-like load is described by $\beta_{j} \in \mathbb{R}$. Furthermore, we consider their masses and lengths to be $m_{p_{j}} \in \mathbb{R}$ and $l_{p_{j}} \in \mathbb{R}$.

\section{A. Kinematic equations}

Based on the position of the chain $\xi=[x z]^{T} \in \mathbb{R}^{2}$ and the angles $\Theta_{j}$ and $\beta_{j}$, the positions of the rotorcrafts $\xi_{r_{i}}$, the links $\xi_{l_{j}}$ and the pendulums $\xi_{p_{j}}$ w.r.t. the inertial frame can be computed as follows:

$$
\begin{aligned}
& \xi_{r_{i}}=\left[\begin{array}{l}
x_{r_{i}} \\
z_{r_{i}}
\end{array}\right]=\xi+\frac{Q_{i} l_{l}}{2}\left[\begin{array}{c}
C_{\Theta_{2}}+V_{i} C_{\Theta_{D_{i}}} \\
-S_{\Theta_{2}}-V_{i} S_{\Theta_{D_{i}}}
\end{array}\right] \in \mathbb{R}^{2} \\
& \xi_{l_{j}}=\left[\begin{array}{l}
x_{l_{j}} \\
z_{l_{j}}
\end{array}\right]=\xi+\frac{E_{i} l_{l}}{2}\left[\begin{array}{c}
C_{\Theta_{2}}+C_{\Theta_{j}} \\
-S_{\Theta_{2}}-S_{\Theta_{j}}
\end{array}\right] \in \mathbb{R}^{2} \\
& \xi_{p_{j}}=\left[\begin{array}{l}
x_{p_{j}} \\
z_{p_{j}}
\end{array}\right]=\xi_{l_{j}}-l_{p_{j}}\left[\begin{array}{l}
S_{\beta_{j}} \\
C_{\beta_{j}}
\end{array}\right] \in \mathbb{R}^{2}
\end{aligned}
$$

where $S_{\bullet}=\sin (\bullet), C_{\bullet}=\cos (\bullet), Q_{k}=\operatorname{sign}(k-2.5), D_{k}=$ $(2 k+1) / 3, E_{k}=k-2$ and $V_{k}=k^{2}-5 k+6$.

By time differentiating the Eqs. (1)-(3), the velocity of each component are obtained, hence:

$$
\begin{aligned}
& \dot{\xi}_{r_{i}}=\dot{\xi}-\frac{Q_{i} l_{l}}{2}\left[\begin{array}{l}
S_{\Theta_{2}} \dot{\Theta}_{2}+V_{i} S_{\Theta_{D_{i}}} \dot{\Theta}_{D_{i}} \\
C_{\Theta_{2}} \dot{\Theta}_{2}+V_{i} C_{\Theta_{D_{i}}} \dot{\Theta}_{D_{i}}
\end{array}\right] \\
& \dot{\xi}_{l_{j}}=\dot{\xi}-\frac{E_{i} l_{l}}{2}\left[\begin{array}{l}
S_{\Theta_{2}} \dot{\Theta}_{2}+S_{\Theta_{j}} \dot{\Theta}_{j} \\
C_{\Theta_{2}} \dot{\Theta}_{2}+C_{\Theta_{j}} \dot{\Theta}_{j}
\end{array}\right] \\
& \dot{\xi}_{p_{j}}=\dot{\xi}_{l_{j}}-l_{p_{j}}\left[\begin{array}{c}
C_{\beta_{j}} \dot{\beta}_{j} \\
-S_{\beta_{j}} \dot{\beta}_{j}
\end{array}\right]
\end{aligned}
$$

The dynamics of the flying multi-link robot is then modelled by the means of the Euler-Lagrange formalism.

\section{B. Dynamics of the ML-UAS}

Let the Lagrangian of the system be expressed as $L=$ $K-U \in \mathbb{R}$ such that $K$ and $U$ stand for the total kinetic and potential energies, respectively. Both terms are defined based on the energies of the quadrotors $\left(K_{r}\right.$ and $\left.U_{r}\right)$, the links $\left(K_{l}\right.$ and $\left.U_{l}\right)$ and the pendulums $\left(K_{p}\right.$ and $\left.U_{p}\right)$, in the manner:

$$
K=K_{r}+K_{l}+K_{p} \in \mathbb{R} \quad ; \quad U=U_{r}+U_{l}+U_{p} \in \mathbb{R}
$$

with

$$
\begin{array}{rlrl}
K_{r} & =\sum_{k=1}^{4}\left\{\frac{m_{r}}{2}\left(\dot{x}_{r_{k}}^{2}+\dot{z}_{r_{k}}^{2}\right)+\frac{I_{r}}{2} \dot{\theta}_{k}^{2}\right\} & U_{r}=g m_{r} \sum_{k=1}^{4} z_{r_{k}} \\
K_{l}=\sum_{k=1}^{3}\left\{\frac{m_{l}}{2}\left(\dot{x}_{l_{k}}^{2}+\dot{z}_{l_{k}}^{2}\right)+\frac{I_{l}}{2} \dot{\Theta}_{k}^{2}\right\} & U_{l}=g m_{l} \sum_{k=1}^{3} z_{l_{k}} \\
K_{p}=\sum_{k=1}^{3} \frac{m_{p_{k}}}{2}\left(\dot{x}_{p_{k}}^{2}+\dot{z}_{p_{k}}^{2}\right) & U_{p}=g \sum_{k=1}^{3} m_{p_{k}} z_{p_{k}}
\end{array}
$$

where $g \in \mathbb{R}$ is the constant of gravity acceleration.

Let the vector of generalized coordinates be defined as $q=\left[\begin{array}{llll}q_{1} & q_{2} & \ldots & q_{5}\end{array}\right]^{T}=\left[\begin{array}{lllll}x & z & \Theta_{1} & \Theta_{2} & \Theta_{3}\end{array}\right]^{T} \in \mathbb{R}^{5}$. By applying the equation provided by the Euler-Lagrange formalism, i.e.

$$
\frac{d}{d t} \frac{\partial}{\partial \dot{q}_{a}} L-\frac{\partial}{\partial q_{a}} L=\tau_{a} \quad \text { with } \quad a=1,2, \ldots, 5
$$

to the Lagrangian, the dynamics of the ML-UAS is provided. It must be noticed that the attitudes of the vehicles are not considered as generalized coordinates since these are used as control inputs.

The equations of motion for the translation of the chain can be expressed as 


$$
\begin{aligned}
& \mu \ddot{x}-\sum_{k=1}^{3}\left\{R_{k} \alpha_{k}\left(S_{\Theta_{k}} \ddot{\Theta}_{k}+C_{\Theta_{k}} \dot{\Theta}_{k}^{2}\right)-\right. \\
& \left.m_{p_{k}} l_{p_{k}}\left(C_{\beta_{k}} \ddot{\beta}_{k}-S_{\beta_{k}} \dot{\beta}_{k}^{2}\right)\right\}=\tau_{x} \\
& \mu(\ddot{z}+g)-\sum_{k=1}^{3}\left\{R_{k} \alpha_{k}\left(C_{\Theta_{k}} \ddot{\Theta}_{k}-S_{\Theta_{k}} \dot{\Theta}_{k}^{2}\right)+\right. \\
& \left.m_{p_{k}} l_{p_{k}}\left(S_{\beta_{k}} \ddot{\beta}_{k}+C_{\beta_{k}} \dot{\beta}_{k}^{2}\right)\right\}=\tau_{z}
\end{aligned}
$$

where $R_{k}=k^{2}-3 k+1$ and $\mu, \alpha_{1}, \alpha_{2}$ and $\alpha_{1} \in \mathbb{R}$ are constants defined as

$$
\begin{aligned}
\mu & =4 m_{r}+3 m_{l}+\sum_{j=1}^{3} m_{p_{j}} & \alpha_{1} & =l_{l}\left(m_{r}+\frac{m_{l}}{2}+\frac{m_{p_{1}}}{2}\right) \\
\alpha_{2} & =\frac{l_{l}}{2}\left(m_{p_{1}}-m_{p_{3}}\right) & \alpha_{3} & =l_{l}\left(m_{r}+\frac{m_{l}}{2}+\frac{m_{p_{3}}}{2}\right)
\end{aligned}
$$

Meanwhile, the equations of motion of the links follow the general form:

$$
\begin{aligned}
& l_{j} \ddot{\Theta}_{j}-R_{j} \alpha_{j}\left(S_{\Theta_{j}} \ddot{x}+C_{\Theta_{j}}(\ddot{z}+g)\right)+ \\
& E_{j}^{2} \frac{l_{l} \alpha_{j}}{2}\left\{C_{\left(\Theta_{j}-\Theta_{2}\right)} \ddot{\Theta}_{2}+S_{\left(\Theta_{j}-\Theta_{2}\right)} \dot{\Theta}_{2}^{2}\right\}+ \\
& E_{j} \eta_{j}\left\{S_{\left(\Theta_{j}-\beta_{j}\right)} \ddot{\beta}_{j}-C_{\left(\Theta_{j}-\beta_{j}\right)} \dot{\beta}_{j}^{2}\right\}+ \\
& \left(1-E_{j}^{2}\right) \sum_{k=1}^{3}\left\{E_{k} \eta_{k}\left\{S_{\left(\Theta_{2}-\beta_{k}\right)} \ddot{\beta}_{k}-C_{\left(\Theta_{2}-\beta_{k}\right)} \dot{\beta}_{k}^{2}\right\}+\right. \\
& \left.\frac{l_{l} \alpha_{k}}{2}\left\{C_{\left(\Theta_{k}-\Theta_{2}\right)} \ddot{\Theta}_{k}-S_{\left(\Theta_{k}-\Theta_{2}\right)} \dot{\Theta}_{k}^{2}\right\}\right\}=\tau_{l_{j}}
\end{aligned}
$$

By applying Eq. (4) and considering the $\beta_{j}$ instead of the generalized coordinates $q_{a}$, the dynamics of the payloads can be represented in the general form:

$$
\begin{aligned}
& m_{p_{j}} l_{p_{j}}^{2} \ddot{\beta}_{j}-m_{p_{j}} l_{p_{j}}\left(C_{\beta_{j}} \ddot{x}-S_{\beta_{j}}(\ddot{z}+g)\right)+ \\
& E_{j} \eta_{j}\left(S_{\left(\Theta_{2}-\beta_{j}\right)} \ddot{\Theta}_{2}+C_{\left(\Theta_{2}-\beta_{j}\right)} \dot{\Theta}_{2}^{2}+\right. \\
& \left.S_{\left(\Theta_{j}-\beta_{j}\right)} \ddot{\Theta}_{j}+C_{\left(\Theta_{j}-\beta_{j}\right)} \dot{\Theta}_{j}^{2}\right)=\tau_{p_{j}}
\end{aligned}
$$

Along the Eqs. (7) and (8), new constants were introduced to reduce the expression, these are:

$$
\begin{aligned}
\eta_{j} & =\frac{m_{p_{j}} l_{p_{j}} l_{l}}{2} \in \mathbb{R} \\
\iota_{1} & =l_{l}^{2}\left(m_{r}+\frac{m_{l}}{4}+\frac{m_{p_{1}}}{4}\right)+I_{l} \in \mathbb{R} \\
\iota_{2} & =l_{l}^{2}\left(m_{r}+\frac{m_{l}}{2}+\frac{m_{p_{1}}}{4}+\frac{m_{p_{3}}}{4}\right)+I_{l} \in \mathbb{R} \\
\iota_{3} & =l_{l}^{2}\left(m_{r}+\frac{m_{l}}{4}+\frac{m_{p_{3}}}{4}\right)+I_{l} \in \mathbb{R}
\end{aligned}
$$

As previously mentioned, the aerial vehicles are the actuators of the system, thus we consider their rotational dynamics

$$
I_{r} \ddot{\theta}_{i}=u_{d_{i}}
$$

where $u_{d_{i}} \in \mathbb{R}$ is the control input of the $i-t h$ vehicle.

\section{Disturbed system}

The system itself is composed by the actuators (MAVs) and the rigid links, in this regard the effects of the payloads can be treated as disturbances, which leads to a nonlinear representation of the system in the form:

$$
\mathbf{M}(q) \ddot{q}+\mathbf{C}(q, \dot{q}) \dot{q}+G(q)=u+\rho
$$

such that

$\mathbf{M}(q)=\left[\begin{array}{ccccc}m_{11} & 0 & \alpha S_{\Theta_{1}} & 0 & -\alpha S_{\Theta_{3}} \\ 0 & m_{22} & \alpha C_{\Theta_{1}} & 0 & -\alpha C_{\Theta_{3}} \\ \alpha S_{\Theta_{1}} & \alpha C_{\Theta_{1}} & m_{33} & m_{34} & 0 \\ 0 & 0 & m_{43} & m_{44} & m_{45} \\ -\alpha S_{\Theta_{3}} & -\alpha C_{\Theta_{3}} & 0 & m_{54} & m_{55}\end{array}\right] \in \mathbb{R}^{5 \times 5}$

with the elements of the matrix being

$$
\begin{aligned}
m_{11}=m_{22} & =4 m_{r}+3 m_{l} \\
m_{33}=m_{55} & =l_{l}^{2}\left(m_{r}+0.25 m_{l}\right)+I_{l} \\
m_{34}=m_{43} & =0.5 l_{l} \alpha_{c} C_{\Theta_{1}-\Theta_{2}} \\
m_{44} & =l_{l}^{2}\left(m_{r}+0.5 m_{l}\right)+I_{l} \\
m_{45}=m_{54} & =0.5 l_{l} \alpha_{c} C_{\Theta_{2}-\Theta_{3}} \\
\alpha & =l_{l}\left(m_{r}+0.5 m_{l}\right)
\end{aligned}
$$

The term $\mathbf{C}(q, \dot{q}) \dot{q}$ in Eq. (9) corresponds to the Coriolis and centripetal effects and it is given as:

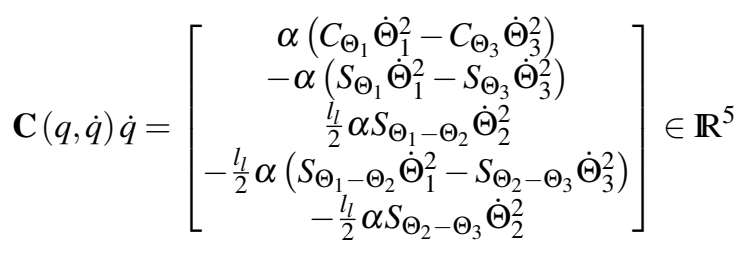

The gravitational effects are comprised in the vector

$$
G(q)=\left[\begin{array}{lllll}
0 & m_{22} g & \alpha g C_{\Theta_{1}} & 0 & -\alpha g C_{\Theta_{3}}
\end{array}\right]^{T} \in \mathbb{R}^{5}
$$

At the right part of Eq. (9), the control input vector $u=$

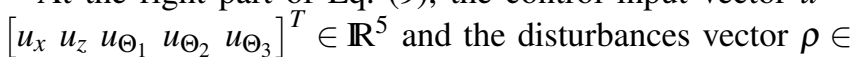
$\mathbb{R}^{5}$ are found.

The perturbations affecting the translational motion are set based on Eqs (5) and (6) as

$$
\begin{aligned}
& \rho_{x}=\sum_{k=1}^{3}\left\{m_{p_{k}} \ddot{x}+m_{p_{k}} l_{p_{k}}\left(C_{\beta_{k}} \ddot{\beta}_{k}-S_{\beta_{k}} \dot{\beta}_{k}^{2}\right)\right\} \\
& \rho_{z}=\sum_{k=1}^{3}\left\{m_{p_{k}}(\ddot{z}+g)-m_{p_{k}} l_{p_{k}}\left(S_{\beta_{k}} \ddot{\beta}_{k}+C_{\beta_{k}} \dot{\beta}_{k}^{2}\right)\right\}
\end{aligned}
$$

For each link motion disturbance, the friction between the corresponding link and the pendulous payload is considered by the addition of the term $\gamma_{j}\left(\dot{\beta}_{j}-\dot{\Theta}_{j}\right)$ where $\gamma_{j} \in \mathbb{R}$ is the friction coefficient. This last consideration implies that the friction effect must be equally considered in the dynamics of the cargos (Eq. (8)) in the term $\tau_{p_{j}}$. 


$$
\begin{aligned}
& \rho_{\Theta_{1}}=-l_{l}^{2} \frac{m_{p_{1}} \ddot{\Theta}_{1}-l_{l} \frac{m_{p_{1}}}{2}\left(S_{\Theta_{1}} \ddot{x}+C_{\Theta_{1}} \ddot{z}\right)-}{} l_{l}^{2} \frac{m_{p_{1}}}{4}\left(C_{\left(\Theta_{1}-\Theta_{2}\right)} \ddot{\Theta}_{2}+S_{\left(\Theta_{1}-\Theta_{2}\right)} \dot{\Theta}_{2}^{2}\right)+ \\
& \eta_{1}\left(S_{\left(\Theta_{1}-\beta_{1}\right)} \ddot{\beta}_{1}-C_{\left(\Theta_{1}-\beta_{1}\right)} \dot{\beta}_{1}^{2}\right)-l_{l} \frac{m_{p_{1}}}{2} g C_{\Theta_{1}}+ \\
& \gamma_{1}\left(\dot{\beta}_{1}-\dot{\Theta}_{1}\right) \\
& \rho_{\Theta_{2}}=-\left(l_{l}^{2}\left(\frac{m_{p_{1}}}{4}+\frac{m_{p_{3}}}{4}\right)\right) \ddot{\Theta}_{2}- \\
&\left(\frac{l_{l}}{2}\left(m_{p_{1}}-m_{p_{3}}\right)\right)\left(S_{\Theta_{2}} \ddot{x}+C_{\Theta_{2}} \ddot{z}\right)- \\
& l_{l}^{2} \frac{m_{p_{1}}}{4}\left(C_{\left(\Theta_{1}-\Theta_{2}\right)} \ddot{\Theta}_{1}-S_{\left(\Theta_{1}-\Theta_{2}\right)} \dot{\Theta}_{1}^{2}\right)- \\
& l_{l}^{2} \frac{m_{p_{3}}}{4}\left(C_{\left(\Theta_{3}-\Theta_{2}\right)} \ddot{\Theta}_{3}-S_{\left(\Theta_{3}-\Theta_{2}\right)} \dot{\Theta}_{3}^{2}\right)+ \\
& \eta_{1}\left(S_{\left(\Theta_{2}-\beta_{1}\right)} \ddot{\beta}_{1}-C_{\left(\Theta_{2}-\beta_{1}\right)} \dot{\beta}_{1}^{2}\right)- \\
& \eta_{3}\left(S_{\left(\Theta_{2}-\beta_{3}\right)} \ddot{\beta}_{3}-C_{\left(\Theta_{2}-\beta_{3}\right)} \dot{\beta}_{3}^{2}\right)- \\
& \frac{l_{l}}{2}\left(m_{p_{1}}-m_{p_{3}}\right) g C_{\Theta_{2}}+\gamma_{2}\left(\dot{\beta}_{2}-\dot{\Theta}_{2}\right) \\
&=-l_{l}^{2} \frac{m_{p_{3}} \ddot{\Theta}_{3}+l_{l} \frac{m_{p_{3}}}{2}\left(S_{\Theta_{3}} \ddot{x}+C_{\Theta_{3}} \ddot{z}\right)-}{4} \\
& l_{l}^{2} \frac{m_{p_{3}}}{4}\left(C_{\left(\Theta_{3}-\Theta_{2}\right)} \ddot{\Theta}_{2}+S_{\left(\Theta_{3}-\Theta_{2}\right)} \dot{\Theta}_{2}^{2}\right)- \\
& \eta_{3}\left(S_{\left(\Theta_{3}-\beta_{3}\right)} \ddot{\beta}_{3}-C_{\left(\Theta_{3}-\beta_{3}\right)} \dot{\beta}_{3}^{2}\right)+l_{l} \frac{m_{p_{3}}}{2} g C_{\Theta_{3}}+ \\
& \gamma_{3}\left(\dot{\beta}_{3}-\dot{\Theta}_{3}\right) \\
&\left.\rho_{\Theta_{3}}\right)
\end{aligned}
$$

The well defined model in Eq. (9) is used in the upcoming section in order to design the AISM controller.

\section{CONTROL}

For control purposes, let the dynamics of the system, based on Eq. (9), be expressed as:

$$
\ddot{q}=f(q, \dot{q})+\mathbf{B}(q) u+w
$$

where $f(q, \dot{q})=f_{o}(q, \dot{q})+\Delta f$ is the dynamic state-dependent function of the system defined by the nominal dynamics $f_{o}(q, \dot{q})=-\mathbf{M}^{-1}(q)(\mathbf{C}(q, \dot{q}) \dot{q}+G(q))$ and the unmodeled uncertainties $\Delta f \in \mathbb{R}^{5}$. The control matrix $\mathbf{B}(q) \in \mathbb{R}^{5 \times 5}$ is composed by the nominal control matrix $\mathbf{B}_{\mathbf{0}}(q)=\mathbf{M}^{-1}(q)$ and the uncertainties in the control matrix $\Delta \mathbf{B} \in \mathbb{R}^{5 \times 5}$ resulting in $\mathbf{B}(q)=\mathbf{B}_{\mathbf{o}}(q)+\Delta \mathbf{B}$. The term $w-\mathbf{M}^{-1}(q) \rho \in \mathbb{R}^{5}$ comprises the external disturbances [12].

According to [12] and [13], we must provide a control input $u=u_{o}+u_{w}$ such that $u_{o} \in \mathbb{R}^{5}$ mitigates the nominal dynamics and $u_{w} \in \mathbb{R}^{5}$ compensates the parametric and environmental uncertainties.

Defining the tracking error vector as $e=q-q^{d} \in \mathbb{R}^{5}$ where the vector $q^{d}$ defines the desired trajectories, we can propose a sliding surface as follows

$$
\sigma=\dot{e}+\lambda e+\varepsilon \in \mathbb{R}^{5}
$$

where $\varepsilon$ is a vector containing the integral terms of the error [7] and $\Lambda=\operatorname{diag}\left(\begin{array}{lllll}\lambda_{x} & \lambda_{z} & \lambda_{\Theta_{1}} & \lambda_{\Theta_{2}} & \lambda_{\Theta_{3}}\end{array}\right) \in \mathbb{R}^{5 \times 5}$ is a diagonal matrix of control gains such that each $\lambda$-gain satisfies $\lambda>0$. The control input must approach $\sigma$ to zero and sustain it there $(\sigma \dot{\sigma}<0)$. Then the nominal control input shall provide stability once the system has reached the sliding surface, $i$, e. $\dot{\sigma}=\mathbf{0} \in \mathbb{R}^{5}$ (with $\mathbf{0}$ the zero vector), i.e:

$$
\mathbf{0}=\ddot{e}+\Lambda \dot{e}+e
$$

By the substitution of the error and its time derivatives, we obtain that

$$
u_{o}=\mathbf{C}(q, \dot{q}) \dot{q}+G(q)+\mathbf{M}(q)\left(\ddot{q}^{d}-\Lambda \dot{e}-e\right)
$$

To mitigate the plant parameter variations and the external disturbances, a viable solution is to consider the control input $u_{w}$ according to the SMC theory as

$$
u_{w}=-\mathbf{M}(q) \mathbf{H} T(\sigma)
$$

such that $\mathbf{H}=\operatorname{diag}\left(\eta_{x} \eta_{z} \eta_{\Theta_{1}} \eta_{\Theta_{2}} \eta_{\Theta_{3}}\right) \in \mathbb{R}^{5 \times 5}$ corresponds to a matrix of adaptive control gains, all subjected to the restriction $\eta>0 \in \mathbb{R}$ and $T=$ $\left[\operatorname{sign} \sigma_{x} \operatorname{sign} \sigma_{z} \ldots \operatorname{sign} \sigma_{\Theta_{3}}\right]^{T} \in \mathbb{R}^{5}$.

Let us recall Eq. (11) and express its time derivative based on the model in Eq. (10) as:

$$
\dot{\sigma}=f_{o}(q, \dot{q})+\mathbf{B}_{\mathbf{0}}(q) u-\ddot{q}^{d}+\Lambda \dot{e}+e+W_{L}
$$

with the term $W_{L}=\Delta f+\Delta \mathbf{B} u+w \in \mathbb{R}^{5}$ being the Lumped uncertainties vector, bounded in the manner

$$
\left\|W_{L}\right\|<\left\|\mathbf{H}^{d}\right\|
$$

where $\mathbf{H}^{d}=\operatorname{diag}\left(\eta_{x}^{d} \eta_{z}^{d} \eta_{\Theta_{1}}^{d} \eta_{\Theta_{2}}^{d} \eta_{\Theta_{3}}^{d}\right) \in \mathbb{R}^{5 \times 5}$ is the terminal value of $\mathbf{H}$.

In order to achieve $\mathbf{H}^{d}$, the dynamics of $\mathbf{H}$ is defined as

$$
\dot{\mathbf{H}}=\mathbf{A}^{-1} \mathbf{S}_{\mathbf{A}} \in \mathbb{R}^{5 \times 5}
$$

with $\mathbf{S}_{\mathbf{A}}=\operatorname{diag}\left(\left|\sigma_{x}\right|\left|\sigma_{z}\right|\left|\sigma_{\Theta_{1}}\right|\left|\sigma_{\Theta_{2}}\right|{ }_{-5}\left|\sigma_{\Theta_{3}}\right|\right) \in \mathbb{R}^{5 \times 5}$ and

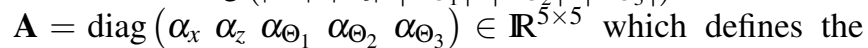
adaptation speed of the $\eta$-gains.

\section{A. Lyapunov stability analysis}

Let us consider the following Lyanpunov candidate function

$$
V=\frac{1}{2} \sigma^{T} \sigma+\frac{1}{2} \mathbf{1}^{T} \tilde{\mathbf{H}}^{T} \mathbf{A} \tilde{\mathbf{H}} \mathbf{1} \in \mathbb{R}
$$

with $\tilde{\mathbf{H}}=\mathbf{H}-\mathbf{H}^{d}$ being the adaptation error and $\mathbf{1} \in \mathbb{R}^{5}$ the ones vector.

By time differentiating Eq. (17) and considering Eqs. (12), (13) and (14), the time derivative of the Lyapunov candidate function can be expressed as

$$
\dot{V}=\sigma^{T}\left(W_{L}-\mathbf{H}^{d} T(\sigma)\right)
$$

and since $\sigma^{T} \mathbf{H}^{d} T(\sigma) \geq 0$ by definition, and considering the boundness property of $W_{L}$ (Eq. (15)), it follows that $\dot{V} \leq 0$ 


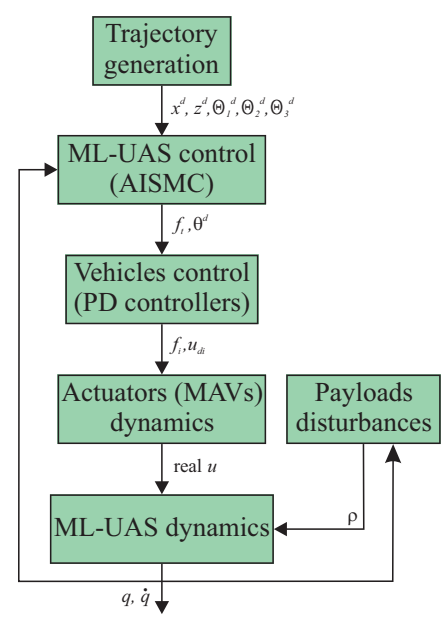

Fig. 2. Control strategy for the ML-UAS

$\forall t>0$ which ensures the asymptotic stability of the system subjected to the proposed control law.

\section{B. MAVs control}

In order to control the attitude of the vehicles, we choose a PD controller given as

$$
u_{d_{i}}=-K_{p_{i}} e_{p_{i}}-K_{v_{i}} e_{v_{i}}
$$

where the constants $K_{p_{i}}, K_{v_{i}} \in \mathbb{R}$ correspond to the proportional and derivative gains, respectively, the position and velocity errors are defined as $e_{p_{i}}=\theta_{i}-\theta_{i}^{d} \in \mathbb{R}$ and $e_{v_{i}}=$ $\dot{\theta}_{i}-\dot{\theta}_{i}^{d} \in \mathbb{R}$ in which the index $d$ stands for the desired position and velocity. The stability of this controller has already been proved in [14].

The desired orientation of the vehicles and the forces to be exerted by each aerial vehicle are computed based on the control inputs generated by the AISM controller.

Let us recall the control input vector in Eq. (9) and the description of the system in Fig. 1, thus

$$
\left[\begin{array}{c}
u_{x} \\
u_{z} \\
u_{\Theta_{1}} \\
u_{\Theta_{2}} \\
u_{\Theta_{3}}
\end{array}\right]=\left[\begin{array}{c}
S_{\theta_{1}} f_{1}+S_{\theta_{2}} f_{2}+S_{\theta_{3}} f_{3}+S_{\theta_{4}} f_{4} \\
C_{\theta_{1}} f_{1}+C_{\theta_{2}} f_{2}+C_{\theta_{3}} f_{3}+C_{\theta_{4}} f_{4} \\
\frac{l_{l}}{2}\left(C_{\Theta_{1}-\theta_{1}} f_{1}-C_{\Theta_{1}-\theta_{2}} f_{2}\right) \\
\frac{l_{l}}{2}\left(C_{\Theta_{2}-\theta_{2}} f_{2}-C_{\Theta_{2}-\theta_{3}} f_{3}\right) \\
\frac{l_{l}}{2}\left(C_{\Theta_{3}-\theta_{3}} f_{3}-C_{\Theta_{3}-\theta_{4}} f_{4}\right)
\end{array}\right]
$$

To avoid the under actuation of the system, let us establish $\forall t>0$ that $\theta^{d}=\theta_{1}^{d}=\theta_{2}^{d}=\theta_{3}^{d}=\theta_{4}^{d} \in \mathbb{R}$ which leads to

$$
\theta^{d}=\tan ^{-1}\left(\frac{u_{x}}{u_{z}}\right)
$$

Therefore the force that each MAV should exert over the system can be computed according to the expression

$$
\left[\begin{array}{l}
f_{1} \\
f_{2} \\
f_{3} \\
f_{4}
\end{array}\right]=\frac{1}{4}\left[\begin{array}{cccc}
1 & 3 & 2 & 1 \\
1 & -1 & 2 & 1 \\
1 & -1 & -2 & 1 \\
1 & -1 & -2 & -3
\end{array}\right]\left[\begin{array}{c}
\sqrt{u_{x}^{2}+u_{z}^{2}} \\
\left(2 u_{\Theta_{1}}\right) /\left(l_{l} C_{\Theta_{1}-\theta^{d}}\right) \\
\left(2 u_{\Theta_{2}}\right) /\left(l_{l} C_{\Theta_{2}-\theta^{d}}\right) \\
\left(2 u_{\Theta_{3}}\right) /\left(l_{l} C_{\Theta_{3}-\theta^{d}}\right)
\end{array}\right]
$$

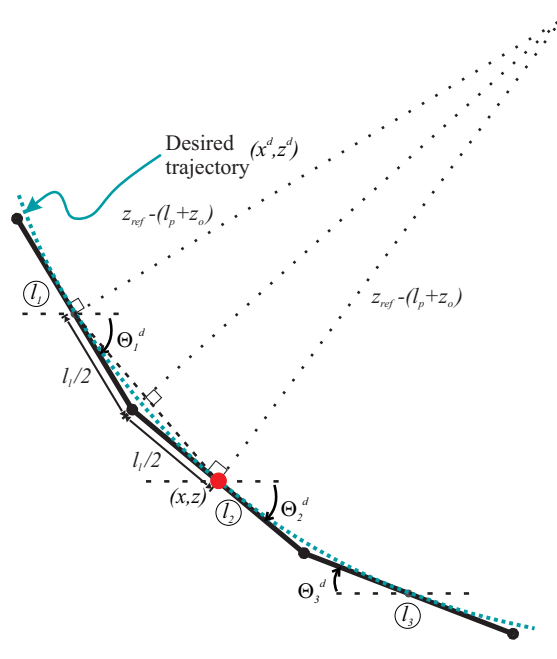

Fig. 3. Desired $\Theta$ angles relations

The control section is summarized in Fig. 2 which shows the control algorithm in a graphical representation for a better understanding. Additionally, the block of trajectory planning can be appreciated thus it is described in the following section.

\section{TRAJECTORY PLANNING AND PICKING STRATEGY}

The trajectory for the altitude of the overall system is based on a co-sinusoidal function whose parameters (frequency and amplitude) are updated based on the object location $\left(x_{o}, z_{o}\right)$ [7]:

The desired trajectory in ${ }^{I} x$ is given in order to keep a constant velocity $v_{h} \in \mathbb{R}$, i. e.

$$
x^{d}(t)=v_{h} t \in \mathbb{R}
$$

Based on this speed pattern, and considering the position in ${ }^{I} x$ of the object $\left(x_{o} \in \mathbb{R}\right)$, we define the grasping time $t_{g} \in \mathbb{R}$ as

$$
t_{g}=\frac{v_{h}}{x_{o}}
$$

For the motion in ${ }^{I} z$, the desired trajectory is defined based on $t_{g}$, the altitude of the chain during the operation $z_{\text {ref }} \in$ $\mathbb{R}$, the length of the pendulums $l_{p}=l_{p_{1}}=l_{p_{2}}=l_{p_{3}}$ and the position of the object in the corresponding axis $z_{o} \in \mathbb{R}$.

$$
z^{d}(t)=z_{\text {ref }}+\left\{z_{\text {ref }}-\left(l_{p}+z_{o}\right)\right\} \cos \left(\frac{\pi}{t_{g}} t\right)
$$

The desired attitude of the links is computed based in a tangent relation w.r.t. the translational pattern. By time differentiation of Eq. (18) and adopting a sign change in the sin function due to the measurement convention, we compute $\Theta_{2}^{d}$ as:

$$
\Theta_{2}^{d}=\tan ^{-1}\left(\left\{z_{\text {ref }}-\left(l_{p}+z_{o}\right)\right\} \frac{\pi}{t_{g}} \sin \left(\frac{\pi}{t_{g}} t\right)\right) \in \mathbb{R}
$$

which, alongside the geometric relations described in Fig. 3, leads to define 

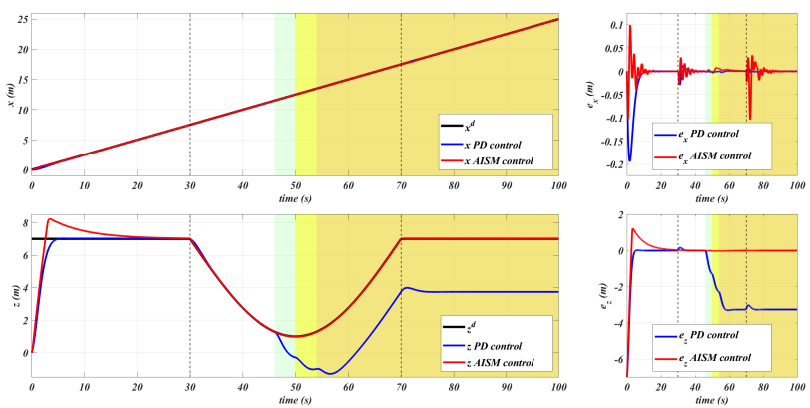

Fig. 4. Translational behavior of the ML-UAS comparison

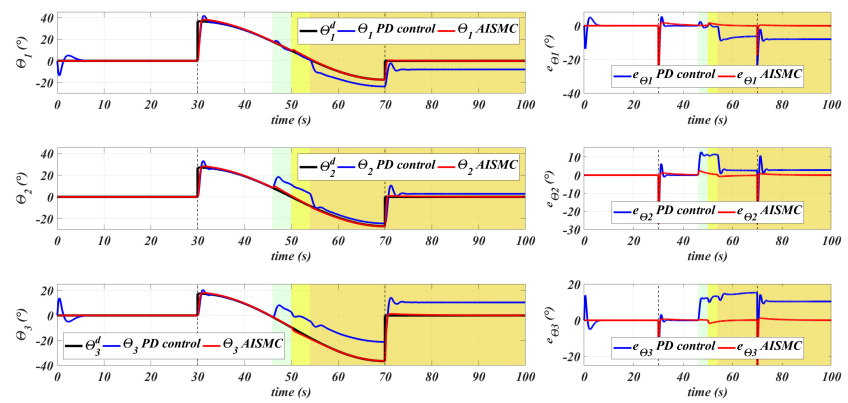

Fig. 5. Rotational links behavior of the ML-UAS comparison

$$
\begin{aligned}
& \Theta_{1}^{d}=\pi-2 \tan ^{-1}\left(\frac{2\left\{z_{\text {ref }}-\left(l_{p}+z_{o}\right)\right\}}{l_{l}}\right)+\Theta_{2}^{d} \in \mathbb{R} \\
& \Theta_{3}^{d}=2 \tan ^{-1}\left(\frac{2\left\{z_{\text {ref }}-\left(l_{p}+z_{o}\right)\right\}}{l_{l}}\right)+\Theta_{2}^{d}-\pi \in \mathbb{R}
\end{aligned}
$$

\section{NUMERICAL SimUlations AND RESUlts}

To validate our control strategy proposal, we developed a numerical simulation with the parameters presented in Table I in which we compared the performance of the system under the command of a PD controller and the AISM controller. The PD control law was defined as

$$
u=-\mathbf{K}_{\mathbf{P}} e-\mathbf{K}_{\mathbf{V}} \dot{e}+\mathbf{M}\left(q^{d}\right) \ddot{q}^{d}+\mathbf{C}\left(q^{d}, \dot{q}^{d}\right) \dot{q}^{d}+G\left(q^{d}\right)
$$

where $\mathbf{K}_{\mathbf{P}}, \mathbf{K}_{\mathbf{V}} \in \mathbb{R}^{5 \times 5}$ are the diagonal control gain matrices of the PD controller to command the overall system.

TABLE I

SIMULATION PARAMETERS

\begin{tabular}{clcl}
\hline Parameter & Value & Parameter & Value \\
\hline$m_{r}$ & $0.62 \mathrm{~kg}$ & $A_{z}$ & $7 \mathrm{~m}$ \\
$I_{r}$ & $0.253 \mathrm{~kg} \mathrm{~m} \mathrm{~m}^{2}$ & $t_{g}$ & $40 \mathrm{~s}$ \\
$m_{l}$ & $0.1 \mathrm{~kg}$ & $v_{h}$ & $0.25 \mathrm{~m} / \mathrm{s}$ \\
$I_{l}$ & $0.0125 \mathrm{~kg} \mathrm{~m} \mathrm{~m}^{2}$ & $x_{o}$ & $12.5 \mathrm{~m}$ \\
$l_{l}$ & $1 \mathrm{~m}$ & $z_{o}$ & $0.5 \mathrm{~m}$ \\
$m_{p_{1}}$ & $0.3 \mathrm{~kg}$ & $\gamma_{j}$ & 0.035 \\
$m_{p_{2}}$ & $0.3 \mathrm{~kg}$ & $g$ & $9.81 \mathrm{~m} / \mathrm{s}^{2}$ \\
$m_{p_{3}}$ & $0.4 \mathrm{~kg}$ & $l_{p}$ & $0.5 \mathrm{~m}$ \\
\hline
\end{tabular}

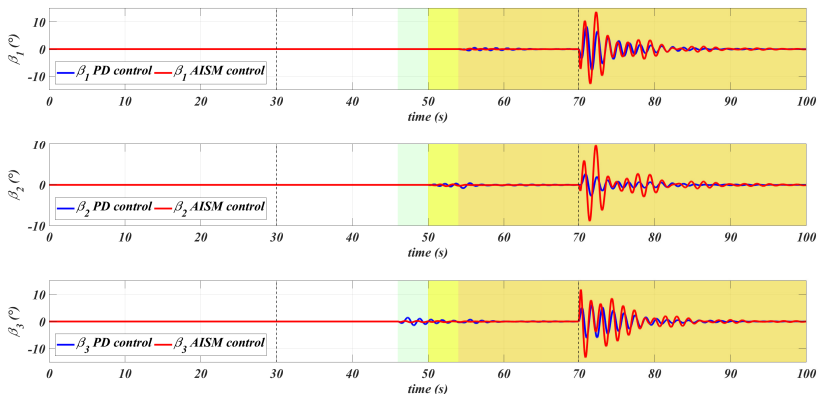

Fig. 6. Payloads motion comparison

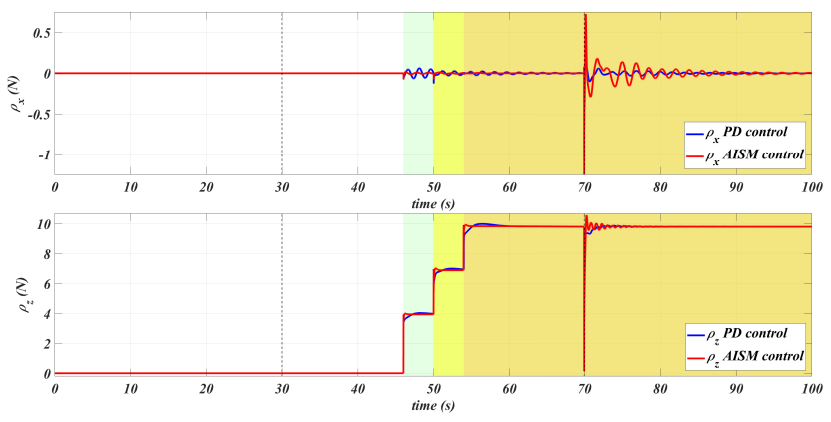

Fig. 7. Translational motion disturbances

The initial conditions of the flying chain were all set to zero as well as the initial attitude of the vehicles.

Moreover, the simulation was divided in 3 different phases described in Table II.

TABLE II

SiMULATION MOTION PHASES

\begin{tabular}{lc}
\hline Phase & Time marks $(s)$ \\
\hline$A_{z}$ and $v_{h}$ reaching phase & $0 \leq t<30$ \\
$\begin{array}{l}\text { Avian inspired trajectory and } \\
\text { picking operation }\end{array}$ & $30 \leq t<70$ \\
Stabilization and $A_{z}$ reaching phase & $70 \leq t$ \\
\hline
\end{tabular}

The results of the simulation are exposed in Figs. 4-9, where the vertical dashed lines in black represents the time marks described in Table II. The three different colors at the background indicates the moment when a payload is picked and transported.

Fig. 4 shows the translational motion of the flying kinematic chain and the corresponding errors. It can be inferred from the ${ }^{I} x$ plot that both controllers drive the system to reach the desired horizontal velocity. It is clear that the motion in ${ }^{I} z$ is successfully tracked by the two controllers until the picking operation starts, in this regard, we can appreciate the robustness of the AISMC.

The motion of the payloads is described in Fig. 6 where the amplitude of the oscillation reaches a larger value in the case of the AISM control than in the application of the PD control.

The movement of the cargos influences directly the dynamics of the overall system as it adds the disturbances previously described in section II. Fig 7 shows the magnitude and 

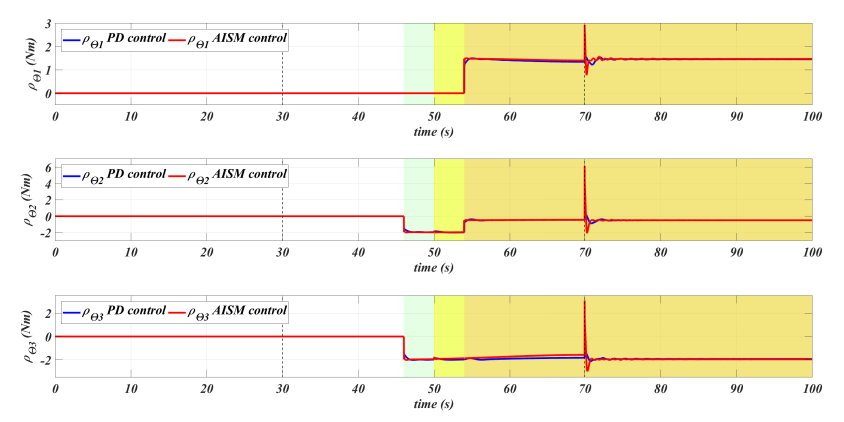

Fig. 8. Rotational links motion disturbances
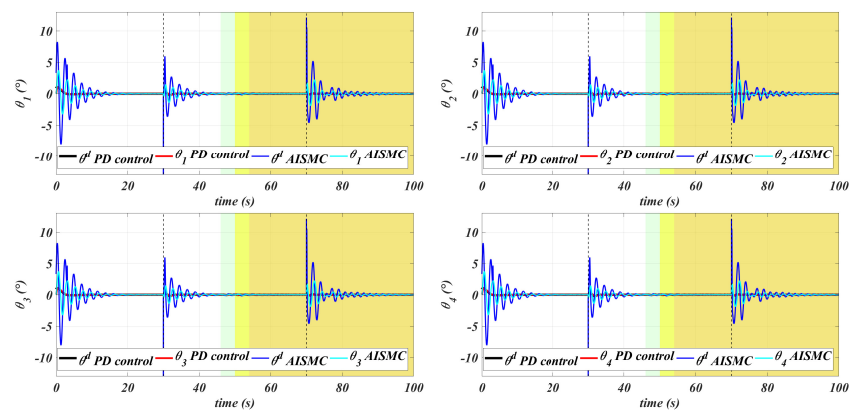

Fig. 9. MAVs behaviour comparison

the behavior of the disturbances produced by the pendulumlike motion of each payload altering the translation of the chain.

The rotational disturbances are shown in Fig. 8 where the disturbances are observed to follow the same behaviour for both cases, differing only in the peak at $t=70 \mathrm{~s}$ presented when applying the AISMC. These peaks imply that the transition is suddenly done and that a modification in the transition strategy needs to be considered in order to have a smoother disturbances influence.

It can be inferred from Fig. 9 that the perturbations are mitigated by the MAVs rotational motion. When implementing the AISMC, the magnitude of the disturbances rises nevertheless the picking pattern is successfully tracked due to the actuators response. In the case of the PD control, no considerable oscillations are present in the motion of the aerial vehicles though the picking trajectory is not followed.

An animation of the simulation is available at https://youtu.be/41Ir6Zuocmg.

\section{CONCLUDING REMARKS AND FUTURE WORK}

In this paper, we have computed the dynamic model of a novel flying kinematic chain, besides, a robust controller based on sliding mode control and adaptive parameters was conceived.

Based on the results obtained from the simulations and the Lyapunov stability analysis, the AISM controller provides stability to the system and disturbances tolerance to accomplish the picking and transport operations.

An improvement of the picking strategy shall be proposed based on a moving objective, in addition the motion of the payloads and the velocity of the operation will be analyze to design an strategy for smooth links transition.

The dynamics of the actuators play an important roll in the performance of the system since so a robust control law will be applied to these MAVs.

The extension to a three dimensional space and the experiments concerning our proposal are conjointly left for future works.

\section{REFERENCES}

[1] Z. Hou, W. Wang, G. Zhang, and C. Han, "A survey on the formation control of multiple quadrotors," in Ubiquitous Robots and Ambient Intelligence (URAI), 2017 14th International Conference on. IEEE, 2017, pp. 219-225.

[2] D. Six, S. Briot, A. Chriette, and P. Martinet, "The kinematics, dynamics and control of a flying parallel robot with three quadrotors," IEEE Robotics and Automation Letters, vol. 3, no. 1, pp. 559-566, 2018.

[3] T. Anzai, M. Zhao, X. Chen, F. Shi, K. Kawasaki, K. Okada, and M. Inaba, "Multilinked multirotor with internal communication system for multiple objects transportation based on form optimization method," in Intelligent Robots and Systems (IROS), 2017 IEEE/RSJ International Conference on. IEEE, 2017, pp. 5977-5984.

[4] M. Zhao, K. Kawasaki, K. Okada, and M. Inaba, "Transformable multirotor with two-dimensional multilinks: modeling, control, and motion planning for aerial transformation," Advanced Robotics, vol. 30, no. 13, pp. 825-845, 2016.

[5] M. Zhao, T. Anzai, F. Shi, X. Chen, K. Okada, and M. Inaba, "Design, modeling, and control of an aerial robot dragon: A dual-rotorembedded multilink robot with the ability of multi-degree-of-freedom aerial transformation," IEEE Robotics and Automation Letters, vol. 3, no. 2, pp. 1176-1183, 2018.

[6] J. Escareno, A. Belbachir, T. Raharijaona, and S. Bouchafa, "Generalized disturbance estimation via eslkf for the motion control of rotorcraft having a rod-suspended load," in Proceedings of the 13th International Conference on Informatics in Control, Automation and Robotics. SCITEPRESS-Science and Technology Publications, Lda, 2016, pp. 526-533.

[7] J. Escareño, J. Castillo, W. Abassi, G. Flores, and K. Camarillo, "Navigation strategy in-flight retrieving and transportation operations for a rotorcraft mav," in 4th Workshop on Research, Education and Development of Unmanned Aerial Systems (RED-UAS 2017), 2017, pp. elec-proc.

[8] K. M. Cabral, S. R. B. dos Santos, S. N. Givigi, and C. L. Nascimento, "Design of model predictive control via learning automata for a single uav load transportation," in Systems Conference (SysCon), 2017 Annual IEEE International. IEEE, 2017, pp. 1-7.

[9] S. Barawkar, M. Radmanesh, M. Kumar, and K. Cohen, "Fuzzy logic based variable damping admittance control for multi-uav collaborative transportation," in 2018 Annual American Control Conference (ACC). IEEE, 2018, pp. 2084-2089.

[10] A. Jafar, S. Fasih-UR-Rehman, S. Fazal-UR-Rehman, and N. Ahmed, "H infinity controller for unmanned aerial vehicle against atmospheric turbulence," American-Eurasian Journal of Scientific Research, vol. 11, no. 4, pp. 305-312, 2016.

[11] G. V. Raffo and M. M. d. Almeida, "A load transportation nonlinear control strategy using a tilt-rotor uav," Journal of Advanced Transportation, vol. 2018, 2018.

[12] Y.-J. Huang, T.-C. Kuo, and S.-H. Chang, "Adaptive sliding-mode control for nonlinear systems with uncertain parameters," Ieee Transactions On Systems, Man And Cybernetics Part B Cybernetics", vol. 38, no. 2, p. 534, 2008.

[13] Y. Pan, C. Yang, L. Pan, and H. Yu, "Integral sliding mode control: performance, modification and improvement," IEEE Transactions on Industrial Informatics, 2017.

[14] J. J. Castillo-Zamora, K. A. Camarillo-Gómez, G. I. Pérez-Soto, and J. Rodríguez-Reséndiz, "Comparison of pd, pid and sliding-mode position controllers for vtail quadcopter stability," IEEE Access, vol. 6, pp. 38 086-38 096, 2018. 